\title{
Stationary resonances of rapidly-rotating Kerr black holes
}

\author{
Shahar Hod \\ The Ruppin Academic Center, Emeq Hefer 40250, Israel \\ and
}

The Hadassah Institute, Jerusalem 91010, Israel

(Dated: February 27, 2018)

\begin{abstract}
The Klein-Gordon equation for a massive scalar field in the background of a rapidly-rotating Kerr black hole is studied analytically. In particular, we derive a simple formula for the stationary (marginally-stable) resonances of the field in the black-hole spacetime. The analytically derived formula is shown to agree with direct numerical computations of the resonances. Our results provide an upper bound on the instability regime of rapidly-rotating Kerr black holes to massive scalar perturbations.
\end{abstract}

\section{INTRODUCTION}

The 'no-hair' conjecture [1,2], put forward by Wheeler more than four decades ago, asserts that stationary black-hole spacetimes should be described by the Kerr-Newman metric. This conjecture therefore suggests that stationary black holes can be characterized by only three externally observable parameters: mass, charge, and angular momentum.

According to the no-hair conjecture, it is expected that static fields (with the exception of the electric field which is associated with a globally conserved charge) cannot survive in the exterior of black holes [1 8]. In particular, such fields are expected to be radiated away to infinity or to be swallowed by the black hole itself [3, 5]. Massless test fields indeed follow this scenario: their relaxation phase in the exterior of black holes is characterized by 'quasinormal ringing', damped oscillations with a discrete spectrum 9, 10] (see also 11] and references therein). These characteristic oscillations are then followed by late-time decaying tails [12, 13].

However, it turns out that (non-static) massive scalar fields 14 can survive in the exterior of rotating black holes due to the well-known phenomena of superradiant scattering [15 27]: a bosonic field of the form $e^{i m \phi} e^{-i \omega t}$ impinging on a rotating Kerr black hole can be amplified as it scatters off the hole if it satisfies the superradiant condition

$$
\omega<m \Omega,
$$

where

$$
\Omega=\frac{a}{2 M r_{+}}
$$

is the angular velocity of the black-hole horizon. Here $M, M a$, and $r_{+}$are the black-hole mass, angular momentum, and horizon-radius, respectively. If in addition the scalar field has a non-zero rest mass, then the mass term (the gravitational attraction between the black hole and the massive field) effectively works as a mirror, preventing the field from escaping to infinity.

In a seminal work, Detweiler [20] studied the Klein-Gordon equation for the black-hole-scalar-field system in the regime $M \mu \ll 1$, that is in the regime where the Compton wavelength of the field is much larger than the length-scale set by the black hole. (Here $\mu \equiv \mathcal{M} G / \hbar c$, where $\mathcal{M}$ is the mass of the field. We shall use natural units in which $G=c=1[28]$.) Using Eqs. (18) and (26) of [20] one finds that marginally stable modes (that is, stationary modes which are characterized by $\Im \omega=0$ ) of the massive scalar field exist for the marginal frequency

$$
\omega=m \Omega
$$

with the discrete spectrum

$$
\mu=m \Omega\left[1+\frac{1}{2}\left(\frac{m M \Omega}{l+1+n}\right)^{2}+O\left[(M \Omega)^{4}\right]\right]
$$

of the field-masses. Here $l$ is the spherical harmonic index of the mode, $m$ is the azimuthal harmonic index with $-l \leq m \leq l$, and $n$ is the resonance parameter which is a non-negative integer [20].

It should be emphasized that the formula (44) is only valid in the regime $M \mu \ll 1$ studied in [20]. Thus, the formula (44) for the field-masses of the stationary resonances is only valid for slowly-rotating black holes (that is, in the regime $M \Omega \ll 1)$. 
The main goal of the present study is to obtain an analytical formula for the field-masses of the stationary resonances in the regime of rapidly-rotating black holes with $a \approx M$ (that is, for $M \Omega \simeq 1 / 2$ ). It is worth mentioning that we have recently obtained a simple upper bound on the field-masses of the stationary (marginally-stable) resonances [26]:

$$
\mu<\sqrt{2} m \Omega .
$$

This upper bound is valid in the entire range $0 \leq a / M \leq 1$ of the dimensionless black-hole spin. Note that the formula (4) above (which is only valid in the $a \ll M$ regime) conforms to this upper bound.

\section{DESCRIPTION OF THE SYSTEM}

The physical system we consider consists of a test scalar field $\Psi$ coupled to a rotating Kerr black hole of mass $M$ and angular-momentum per unit mass $a$. In Boyer-Lindquist coordinates $(t, r, \theta, \phi)$ the spacetime metric is given by $[29,30]$

$$
d s^{2}=-\left(1-\frac{2 M r}{\rho^{2}}\right) d t^{2}-\frac{4 M a r \sin ^{2} \theta}{\rho^{2}} d t d \phi+\frac{\rho^{2}}{\Delta} d r^{2}+\rho^{2} d \theta^{2}+\left(r^{2}+a^{2}+\frac{2 M a^{2} r \sin ^{2} \theta}{\rho^{2}}\right) \sin ^{2} \theta d \phi^{2},
$$

where $\Delta \equiv r^{2}-2 M r+a^{2}$ and $\rho \equiv r^{2}+a^{2} \cos ^{2} \theta$. The black-hole (event and inner) horizons are located at the zeroes of $\Delta$ :

$$
r_{ \pm}=M \pm\left(M^{2}-a^{2}\right)^{1 / 2}
$$

We shall henceforth assume that the black hole is rapidly-rotating (near-extremal) with $a \approx M$.

The dynamics of a massive scalar field $\Psi$ in the Kerr spacetime is governed by the Klein-Gordon equation [31]

$$
\left(\nabla^{a} \nabla_{a}-\mu^{2}\right) \Psi=0
$$

One may decompose the field as

$$
\Psi_{l m}(t, r, \theta, \phi)=e^{i m \phi} S_{l m}(\theta ; a \omega) R_{l m}(r ; a \omega) e^{-i \omega t},
$$

where $\omega$ is the (conserved) frequency of the mode. (We shall henceforth omit the indices $l$ and $m$ for brevity.) With the decomposition (9), $R$ and $S$ obey radial and angular equations both of confluent Heun type coupled by a separation constant $K(a \omega)[31-36]$.

The angular functions $S(\theta ; a \omega)$ are the spheroidal harmonics which are solutions of the angular equation [31 36]

$$
\frac{1}{\sin \theta} \frac{\partial}{\partial \theta}\left(\sin \theta \frac{\partial S}{\partial \theta}\right)+\left[K+a^{2}\left(\mu^{2}-\omega^{2}\right)-a^{2}\left(\mu^{2}-\omega^{2}\right) \cos ^{2} \theta-\frac{m^{2}}{\sin ^{2} \theta}\right] S=0 .
$$

The angular functions are required to be regular at the poles $\theta=0$ and $\theta=\pi$. These boundary conditions pick out a discrete set of eigenvalues $\left\{K_{l m}\right\}$ labeled by the integers $l$ and $m$. For $a^{2}\left(\mu^{2}-\omega^{2}\right) \lesssim m^{2}$ one can treat $a^{2}\left(\omega^{2}-\mu^{2}\right) \cos ^{2} \theta$ in Eq. (10) as a perturbation term on the generalized Legendre equation and obtain the perturbation expansion [34]

$$
K_{l m}+a^{2}\left(\mu^{2}-\omega^{2}\right)=l(l+1)+\sum_{k=1}^{\infty} c_{k} a^{2 k}\left(\mu^{2}-\omega^{2}\right)^{k}
$$

for the separation constants $K_{l m}$. The expansion coefficients $\left\{c_{k}(l, m)\right\}$ are given in Ref. [34].

The radial Teukolsky equation is given by [31, 35]

$$
\Delta \frac{d}{d r}\left(\Delta \frac{d R}{d r}\right)+\left[H^{2}+\Delta\left[2 m a \omega-K-\mu^{2}\left(r^{2}+a^{2}\right)\right]\right] R=0
$$

where $H \equiv\left(r^{2}+a^{2}\right) \omega-a m$. We are interested in solutions of the radial equation (12) with the physical boundary conditions of purely ingoing waves at the black-hole horizon (as measured by a comoving observer) and a bounded (decaying) solution at spatial infinity [18 27]. That is,

$$
R \sim \begin{cases}\frac{1}{r} e^{-\sqrt{\mu^{2}-\omega^{2}} y} & \text { as } r \rightarrow \infty \quad(y \rightarrow \infty) \\ e^{-i(\omega-m \Omega) y} & \text { as } r \rightarrow r_{H} \quad(y \rightarrow-\infty)\end{cases}
$$

where the "tortoise" radial coordinate $y$ is defined by $d y=\left[\left(r^{2}+a^{2}\right) / \Delta\right] d r$.

Note that a bound state (a state decaying exponentially at spatial infinity) is characterized by $\omega^{2}<\mu^{2}$. The boundary conditions (13) single out a discrete set of complex resonances $\left\{\omega_{n}(\mu)\right\}$ which correspond to the bound states of the massive field [18 27, 37, 38]. The stationary (marginally-stable) resonances, which are the solutions we are interested in in this paper, are characterized by $\Im \omega=0$. 


\section{THE STATIONARY SCALAR RESONANCES}

As we shall now show, the field (9) with the marginal frequency (3) describes a stationary resonance of the KleinGordon equation (8) in the black-hole spacetime. In particular, we shall now derive an analytical formula for the discrete spectrum $\{M \mu(m, l, n)\}$ of field-masses which satisfy the stationary resonance condition $\Im \omega=0$. To that end, it is convenient to define new dimensionless variables

$$
x \equiv \frac{r-r_{+}}{r_{+}} ; \tau \equiv 8 \pi M T_{B H}=\frac{r_{+}-r_{-}}{r_{+}} ; k \equiv 2 m \Omega r_{+} ; \epsilon \equiv \sqrt{\mu^{2}-(m \Omega)^{2}} r_{+} ，
$$

in terms of which the radial equation (12) becomes

$$
x(x+\tau) \frac{d^{2} R}{d x^{2}}+(2 x+\tau) \frac{d R}{d x}+V R=0,
$$

where $V \equiv H^{2} / r_{+}^{2} x(x+\tau)-K_{l m}+2 m^{2} a \Omega-\mu^{2}\left[r_{+}^{2}(x+1)^{2}+a^{2}\right]$ and $H=k r_{+} x\left(\frac{1}{2} x+1\right)$.

We first consider the radial equation (15) in the far region $x \gg \tau$. Then Eq. (15) is well approximated by

$$
x^{2} \frac{d^{2} R}{d x^{2}}+2 x \frac{d R}{d x}+V_{\mathrm{far}} R=0
$$

where $V_{\text {far }}=-(\epsilon x)^{2}+k^{2} x / 2+\left[-K_{l m}+2 m^{2} a \Omega+k^{2}-\mu^{2}\left(r_{+}^{2}+a^{2}\right)\right]$. A solution of Eq. (16) that satisfies the boundary condition (13) can be expressed in terms of the confluent hypergeometric functions $M(a, b, z)$ [34, 39]

$$
R=C_{1}(2 \epsilon)^{\frac{1}{2}+\beta} x^{-\frac{1}{2}+\beta} e^{-\epsilon x} M\left(\frac{1}{2}+\beta-\kappa, 1+2 \beta, 2 \epsilon x\right)+C_{2}(\beta \rightarrow-\beta),
$$

where $C_{1}$ and $C_{2}$ are constants. Here

$$
\beta^{2} \equiv K_{l m}+\frac{1}{4}+\mu^{2}\left(r_{+}^{2}+a^{2}\right)-k^{2}-2 m^{2} a \Omega
$$

and

$$
\kappa \equiv \frac{\frac{1}{4} k^{2}-\epsilon^{2}}{\epsilon}
$$

The notation $(\beta \rightarrow-\beta)$ means "replace $\beta$ by $-\beta$ in the preceding term."

We next consider the near horizon region $x \ll 1$. The radial equation is given by Eq. (15) with $V \rightarrow V_{\text {near }} \equiv$ $-K_{l m}+2 m^{2} a \Omega-\mu^{2}\left(r_{+}^{2}+a^{2}\right)+k^{2} x /(x+\tau)$. The physical solution obeying the ingoing boundary condition at the horizon is given by [34, 39]

$$
R=\left(\frac{x}{\tau}+1\right)_{2}^{-i k} F_{1}\left(\frac{1}{2}+\beta-i k, \frac{1}{2}-\beta-i k ; 1 ;-x / \tau\right),
$$

where ${ }_{2} F_{1}(a, b ; c ; z)$ is the hypergeometric function.

The solutions (17) and (20) can be matched in the overlap region $\tau \ll x \ll 1$. The $x \ll 1$ limit of Eq. (17) yields [34, 39]

$$
R \rightarrow C_{1}(2 \epsilon)^{\frac{1}{2}+\beta} x^{-\frac{1}{2}+\beta}+C_{2}(\beta \rightarrow-\beta) .
$$

The $x \gg \tau$ limit of Eq. (20) yields [34, 39]

$$
R \rightarrow \tau^{\frac{1}{2}-\beta} \frac{\Gamma(2 \beta)}{\Gamma\left(\frac{1}{2}+\beta-i k\right) \Gamma\left(\frac{1}{2}+\beta+i k\right)} x^{-\frac{1}{2}+\beta}+(\beta \rightarrow-\beta) .
$$

By matching the two solutions in the overlap region one finds

$$
C_{1}=\tau^{\frac{1}{2}-\beta} \frac{\Gamma(2 \beta)}{\Gamma\left(\frac{1}{2}+\beta-i k\right) \Gamma\left(\frac{1}{2}+\beta+i k\right)}(2 \epsilon)^{-\frac{1}{2}-\beta},
$$

and

$$
C_{2}=\tau^{\frac{1}{2}+\beta} \frac{\Gamma(-2 \beta)}{\Gamma\left(\frac{1}{2}-\beta-i k\right) \Gamma\left(\frac{1}{2}-\beta+i k\right)}(2 \epsilon)^{-\frac{1}{2}+\beta}
$$


Approximating Eq. (17) for $x \rightarrow \infty$ one gets [34, 39]

$$
\begin{aligned}
R \rightarrow & {\left[C_{1}(2 \epsilon)^{-\kappa} \frac{\Gamma(1+2 \beta)}{\Gamma\left(\frac{1}{2}+\beta-\kappa\right)} x^{-1-\kappa}+C_{2}(\beta \rightarrow-\beta)\right] e^{\epsilon x} } \\
& +\left[C_{1}(2 \epsilon)^{\kappa} \frac{\Gamma(1+2 \beta)}{\Gamma\left(\frac{1}{2}+\beta+\kappa\right)} x^{-1+\kappa}(-1)^{-\frac{1}{2}-\beta+\kappa}+C_{2}(\beta \rightarrow-\beta)\right] e^{-\epsilon x} .
\end{aligned}
$$

A bound state is characterized by a decaying field at spatial infinity. The coefficient of the growing exponent $e^{\epsilon x}$ in Eq. (25) should therefore vanish. Taking cognizance of Eqs. (23)-(25), one finds the characteristic equation

$$
\frac{1}{\Gamma\left(\frac{1}{2}+\beta-\kappa\right)}=\left[\frac{\Gamma(-2 \beta)}{\Gamma(2 \beta)}\right]^{2} \frac{\Gamma\left(\frac{1}{2}+\beta-i k\right) \Gamma\left(\frac{1}{2}+\beta+i k\right)}{\Gamma\left(\frac{1}{2}-\beta-i k\right) \Gamma\left(\frac{1}{2}-\beta+i k\right) \Gamma\left(\frac{1}{2}-\beta-\kappa\right)}(2 \epsilon \tau)^{2 \beta}
$$

for the stationary bound states of the massive scalar field. Note that the r.h.s. of Eq. (26) is of order $O\left(\tau^{2 \beta}\right) \ll 1$. Thus, using the well-known pole structure of the Gamma functions [34], one finds that the resonance condition (26) can be written as

$$
\frac{1}{2}+\beta-\kappa=-n+O\left(\tau^{2 \beta}\right)
$$

where $n \geq 0$ is a non-negative integer.

We shall henceforth assume that $2 \beta>1$ [40] and expand all quantities to first order in the small parameter $\tau$. Taking cognizance of Eqs. (11), (14), (18), and (19), one finds

$$
\beta^{2}=\left(l+\frac{1}{2}\right)^{2}-\frac{3}{2} m^{2}+\frac{1}{4} m^{2} \tau+O\left(\tau^{2}, \epsilon^{2}\right)
$$

and

$$
\kappa=\frac{m^{2}}{4 \epsilon}-\epsilon+O\left(\tau^{2}\right) .
$$

Substituting Eqs. (28)-(29) into (27), one finds that the resonance condition for the stationary modes can be expressed as a polynomial equation for the dimensionless variable $\epsilon$ :

$$
4\left[(2 l+1)^{2}-m^{2}(4-\tau)-(2 n+1)^{2}\right] \epsilon^{2}+4 m^{2}(2 n+1) \epsilon-m^{4}+O\left(\tau^{2 \alpha}, \epsilon^{3}\right)=0,
$$

where $\alpha \equiv \min \{1, \beta\}$. This simple equation can easily be solved to yield [41]

$$
\bar{\epsilon}(l, m, n) \equiv \frac{\epsilon}{m}=\frac{m}{2(\ell+1+2 n)}-\frac{m^{3}}{4 \ell(\ell+1+2 n)^{2}} \tau+O\left(\tau^{2 \alpha}, \bar{\epsilon}^{3}\right),
$$

where $\ell \equiv \sqrt{(2 l+1)^{2}-4 m^{2}}[42]$. The field-masses of the stationary resonances are given by $\mu=\sqrt{(m \Omega)^{2}+\left(\epsilon / r_{+}\right)^{2}}$ [see Eq. (14)], which implies

$$
\mu=m \Omega\left[1+2 \bar{\epsilon}^{2}+O\left(\tau^{2}, \bar{\epsilon}^{4}\right)\right]
$$

\section{NUMERICAL CONFIRMATION}

We shall now test the accuracy of the analytically derived formula (32) for the field-masses of the stationary resonances. The stationary resonances can be computed using standard numerical techniques, see [23, 27] for details. In Table \ we present a comparison between the analytically derived field-masses of the stationary resonances (32), and the numerically computed field-masses [23, 27] for the physically most interesting mode [18 27], $l=m=1$ with $n=0$. We find an almost perfect agreement between the two in the $\tau \ll 1(a / M \gtrsim 0.99)$ regime. In fact, one finds that the agreement between the numerical data and the analytical formula (32) is quite good already at $a / M=0.9$. This is quite surprising since the assumption $\tau \ll 1$ breaks down for this value of the dimensionless spin parameter. 


\begin{tabular}{|c|c|c|c|c|c|c|}
\hline$a / M$ & 0.9 & 0.95 & 0.99 & 0.995 & 0.999 & 1.0 \\
\hline$\mu_{\text {ana }} / \mu_{\text {num }}$ & 1.029 & 1.024 & 1.007 & 1.006 & 1.004 & 1.003 \\
\hline
\end{tabular}

TABLE I: Stationary resonances of a massive scalar field in the background of a rapidly-rotating Kerr black hole. The data shown is for the fundamental mode $l=m=1$ with $n=0$, see also [23, 27]. We display the ratio between the analytically

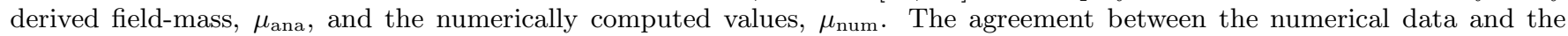
analytical formula (32) is better than $3 \%$ in the $a / M \gtrsim 0.9$ regime. (In fact, the agreement becomes much better than $1 \%$ in the $a / M \gtrsim 0.99$ regime).

\section{SUMMARY}

In summary, we have analyzed the spectrum of stationary (marginally-stable) resonances of massive scalar fields in the spacetime of rapidly-rotating (near-extremal) Kerr black holes. In particular, we have obtained an analytical expression for the field-masses of the stationary $(\Im \omega=0)$ resonances, see Eqs. (31)-(32). We have shown that the analytically derived formula agrees with direct numerical computations of the resonances. Finally, we note that our results [compare Eqs. (5) and (32)] provide an improved upper bound on the instability regime of rapidly-rotating Kerr black holes to massive scalar perturbations.

\section{ACKNOWLEDGMENTS}

This research is supported by the Carmel Science Foundation. I thank Yael Oren, Arbel M. Ongo and Ayelet B. Lata for stimulating discussions.

[1] R. Ruffini and J. A. Wheeler, Phys. Today 24, 30 (1971).

[2] B. Carter, in Black Holes, Proceedings of 1972 Session of Ecole d'ete de Physique Theorique, edited by C. De Witt and B. S. De Witt (Gordon and Breach, New York, 1973).

[3] J. D. Bekenstein, Phys. Today 33, 24 (1980).

[4] D. Núñez, H. Quevedo, and D. Sudarsky, Phys. Rev. Lett. 76, 571 (1996).

[5] S. Hod, Phys. Rev. D 84, 124030 (2011) arXiv:1112.3286].

[6] J. E. Chase, Commun. Math. Phys. 19, 276 (1970); J. D. Bekenstein, Phys. Rev. Lett. 28, 452 (1972); C. Teitelboim, Lett. Nuovo Cimento 3, 326 (1972); I. Pena and D. Sudarsky, Class. Quant. Grav. 14, 3131 (1997).

[7] J. D. Bekenstein, Phys. Rev. D 5, 1239 (1972); 5, 2403 (1972); M. Heusler, J. Math. Phys. 33, 3497 (1992); D. Sudarsky, Class. Quantum Grav. 12, 579 (1995).

[8] J. Hartle, Phys. Rev. D 3, 2938 (1971); C. Teitelboim, Lett. Nuovo Cimento 3, 397 (1972).

[9] H. P. Nollert, Class. Quantum Grav. 16, R159 (1999).

[10] E. Berti, V. Cardoso and A. O. Starinets, Class. Quant. Grav. 26, 163001 (2009).

[11] E. W. Leaver, Proc. R. Soc. A 402, 285 (1985); B. Mashhoon, Phys. Rev. D 31, 290 (1985); H. P. Nollert, Phys. Rev. D 47, 5253 (1993); S. Hod, Phys. Rev. Lett. 81, 4293 (1998) arXiv:gr-qc/9812002; G. T. Horowitz and V. E. Hubeny, Phys. Rev. D 62, 024027 (2000); K. Glampedakis and N. Andersson, Phys. Rev. D 64, 104021 (2001); U. Keshet and S. Hod, Phys. Rev. D 76, R061501 (2007) arXiv:0705.1179]; S. Hod, Phys. Rev. D 75, 064013 (2007) arXiv:gr-qc/0611004; S. Hod, Class. and Quant. Grav. 24, 4235 (2007) arXiv:0705.2306]; A. Gruzinov, arXiv:gr-qc/0705.1725; A. Pesci, Class. Quantum Grav. 24, 6219 (2007); S. Hod, Phys. Rev. D 78, 084035 (2008) arXiv:0811.3806; S. Hod, Phys. Lett. B 666 483 (2008) arXiv:0810.5419; S. Hod, Phys. Rev. D 80, 064004 (2009) arXiv:0909.0314; V. Cardoso, A. S. Miranda, E. Berti, H. Witek, and V. T. Zanchin, Phys. Rev. D 79, 064016 (2009); S. Hod, Phys. Lett. A 374, 2901 (2010) arXiv:1006.4439; R. A. Konoplya and A. Zhidenko, Rev. Mod. Phys. 83, 793 (2011); S. Hod, Phys. Rev. D. 84, 044046 (2011) arXiv:1109.4080; Y. Décanini, A. Folacci, and B. Raffaelli, Phys. Rev. D 84, 084035 (2011); S. Hod, Phys. Lett. B 710, 349 (2012) arXiv:1205.5087]; S. Hod, Phys. Lett. B 715, 348 (2012) arXiv:1207.5282.

[12] R. H. Price, Phys. Rev. D 5, 2419 (1972); C. Gundlach, R. H. Price, and J. Pullin, Phys. Rev. D 49, 883 (1994); J. Bicák, Gen. Relativ. Gravitation 3, 331 (1972).

[13] E. S. C. Ching, P. T. Leung, W. M. Suen, and K. Young, Phys. Rev. Lett. 74, 2414 (1995); E. S. C. Ching, P. T. Leung, W. M. Suen, and K. Young, Phys. Rev. D 52, 2118 (1995); S. Hod and T. Piran, Phys. Rev. D 58, 024017 (1998) arXiv:gr-qc/9712041; S. Hod and T. Piran, Phys. Rev. D 58, 024018 (1998) arXiv:gr-qc/9801001]; S. Hod and T. Piran, Phys. Rev. D 58, 044018 (1998) arXiv:gr-qc/9801059; S. Hod and T. Piran, Phys. Rev. D 58, 024019 (1998) arXiv:gr-qc/9801060; S. Hod, Phys. Rev. D 58, 104022 (1998) arXiv:gr-qc/9811032; S. Hod, Phys. Rev. D 61, 024033 (2000) arXiv:gr-qc/9902072]; S. Hod, Phys. Rev. D 61, 064018 (2000) arXiv:gr-qc/9902073]; L. Barack, Phys. Rev. D 61, 024026 (2000); S. Hod, Phys. Rev. Lett. 84, 10 (2000) arXiv:gr-qc/9907096; S. Hod, Phys. Rev. D 60, 104053 (1999) arXiv:gr-qc/9907044; S. Hod, Class. Quant. Grav. 26, 028001 (2009) arXiv:0902.0237; S. Hod, Class. Quant. Grav. 18, 1311 (2001) arXiv:gr-qc/0008001; S. Hod, Phys. Rev. D 66, 024001 (2002) arXiv:gr-qc/0201017]; R. J. Gleiser, R. 
H. Price, and J. Pullin, Class. Quant. Grav. 25, 072001 (2008); M. Tiglio, L. E. Kidder, and S. A. Teukolsky, Class. Quant. Grav. 25, 105022 (2008); R. Moderski and M. Rogatko, Phys. Rev. D 77, 124007 (2008); X. He and J. Jing, Nucl. Phys.B 755, 313 (2006); H. Koyama and A. Tomimatsu, Phys. Rev. D 65, 084031 (2002); B. Wang, C. Molina, and E. Abdalla, Phys. Rev. D 63, 084001 (2001); A. Zenginoglu and M. Tiglio, Phys. Rev.D 80, 024044 (2009); A. J. Amsel, G. T. Horowitz, D. Marolf, and M. M. Roberts, J. High Energy Phys. 0909:044 (2009).

[14] This statement seems to hold true for all bosonic fields.

[15] Ya. B. Zel'dovich, Pis'ma Zh. Eksp. Teor. Fiz. 14, 270 (1971) [JETP Lett. 14, 180 (1971)]; Zh. Eksp. Teor. Fiz. 62, 2076 (1972) [Sov. Phys. JETP 35, 1085 (1972)].

[16] W. H. Press and S. A. Teukolsky, Astrophys. J. 185, 649 (1973).

[17] W. H. Press and S. A. Teukolsky, Nature 238, 211 (1972).

[18] T. Damour, N. Deruelle and R. Ruffini, Lett. Nuovo Cimento 15, 257 (1976).

[19] T. M. Zouros and D. M. Eardley, Annals of physics 118, 139 (1979).

[20] S. Detweiler, Phys. Rev. D 22, 2323 (1980).

[21] H. Furuhashi and Y. Nambu, Prog. Theor. Phys. 112, 983 (2004).

[22] V. Cardoso, O. J. C. Dias, J. P. S. Lemos and S. Yoshida, Phys. Rev. D 70, 044039 (2004); Erratum-ibid. D 70, 049903 (2004); V. Cardoso and J. P. S. Lemos, Phys. Lett. B 621, 219 (2005); V. Cardoso and S. Yoshida, JHEP 0507:009 (2005); H. Witek, V. Cardoso, A. Ishibashi, and U. Sperhake, e-print arXiv:1212.0551.

[23] S. R. Dolan, Phys. Rev. D 76, 084001 (2007).

[24] S. Hod and O. Hod, Phys. Rev. D 81, Rapid communication 061502 (2010) arXiv:0910.0734; S. Hod and O. Hod, e-print arXiv:0912.2761.

[25] H. R. Beyer, J. Math. Phys. 52, 102502 (2011).

[26] S. Hod, Phys. Lett. B 708, 320 (2012) arXiv:1205.1872; S. Hod, Phys. Lett. B 713, 505 (2012); S. Hod, Phys. Rev. D 86, 104026 (2012) arXiv:1211.3202; S. Hod, Phys. Lett. B 718, 1489 (2013).

[27] S. R. Dolan, e-print arXiv:1212.1477.

[28] In these units $\mu$ has the dimensions of $1 /$ length.

[29] S. Chandrasekhar, The Mathematical Theory of Black Holes, (Oxford University Press, New York, 1983).

[30] R. P. Kerr, Phys. Rev. Lett. 11, 237 (1963).

[31] S. A. Teukolsky, Phys. Rev. Lett. 29, 1114 (1972); S. A. Teukolsky, Astrophys. J. 185, 635 (1973).

[32] A. Ronveaux, Heun's differential equations. (Oxford University Press, Oxford, UK, 1995); C. Flammer, Spheroidal Wave Functions (Stanford University Press, Stanford, 1957).

[33] P. P. Fiziev, e-print arXiv:0902.1277; R. S. Borissov and P. P. Fiziev, e-print arXiv:0903.3617; P. P. Fiziev, Phys. Rev. D 80, 124001 (2009); P. P. Fiziev, Class. Quant. Grav. 27, 135001 (2010).

[34] M. Abramowitz and I. A. Stegun, Handbook of Mathematical Functions (Dover Publications, New York, 1970).

[35] T. Hartman, W. Song, and A. Strominger, JHEP 1003:118 (2010); S. Hod, Phys. Rev. Lett. 100, 121101 (2008) arXiv:0805.3873.

[36] S. Hod, Phys. Lett. B 717, 462 (2012).

[37] We note that, in addition to the bound states of the massive field, the field also has an infinite set of discrete quasinormal resonances [38] which are characterized by outgoing waves at spatial infinity.

[38] L. E. Simone and C. M. Will, Class. Quantum Grav. 9, 963 (1992).

[39] P. M. Morse and H. Feshbach, Methods of Theoretical Physics (McGraw-Hill, New York, 1953).

[40] In the $\tau \ll 1$ regime the condition $2 \beta>1$ is satisfied by modes with $m<\sqrt{2 l(l+1) / 3}$, see Eq. (28) below. In particular, it is satisfied by the fundamental $l=m=1$ mode.

[41] We denote by $\epsilon$ the solution of the quadratic equation (30). The $O\left(\epsilon^{3}\right)$ term in (30) is $-16(2 n+1) \epsilon^{3}$. In the $\tau \rightarrow 0$ limit this term introduces a small correction of the form: $\epsilon \rightarrow \epsilon(1+\delta)$ with $\delta \simeq \frac{m^{2}(2 n+1)}{\ell(\ell+1+2 n)^{2}}$. Note that in the regime $2 \beta>1$ that we consider $(m<\sqrt{2 l(l+1) / 3}$, see [40] $)$, one has $\delta<\frac{\frac{2}{3} l(l+1)}{\sqrt{\frac{4}{3} l(l+1)+1}\left[\sqrt{\frac{4}{3} l(l+1)+1}+1\right]^{2}} \ll 1$.

[42] Note that $\bar{\epsilon}^{2} \ll 1$ in the regime $2 \beta>1$ that we consider $(m<\sqrt{2 l(l+1) / 3}$, see [40] $)$. This justifies the perturbation expansion (11). 